\title{
Directed rehabilitation reduces pain and depression while increasing independence and satisfaction with life for patients with paraplegia due to epidural metastatic spinal cord compression
}

\author{
Robert L. Ruff, MD, PhD; ${ }^{1-2 *}$ Van W. Adamson, MD; ${ }^{1-2}$ Suzanne S. Ruff, PhD; ${ }^{1}$ Xiaofeng Wang, $\mathbf{P h D}^{\mathbf{3}}$ \\ ${ }^{1}$ Neurology and Spinal Cord Injury and Dysfunction Services, Louis Stokes Cleveland Department of Veterans Affairs \\ Medical Center, Cleveland, OH; ${ }^{2}$ Department of Neurology, Case Western Reserve University, Cleveland, $\mathrm{OH}$; \\ ${ }^{3}$ Department of Quantitative Health Sciences, The Cleveland Clinic, Cleveland, $\mathrm{OH}$
}

\begin{abstract}
We determined whether directed rehabilitation affected survival, pain, depression, independence, and satisfaction with life for veterans who were nonambulatory after spinal epidural metastasis (SEM) treatment. We compared 12 consecutive paraplegic veterans who received 2 weeks of directed rehabilitation with a historical control group of 30 paraplegic veterans who did not receive rehabilitation. The rehabilitation program emphasized transfers, bowel and bladder care, incentive spirometry, nutrition, and skin care. The outcome measures were survival, independence, pain levels, depression, and satisfaction with life. Patients receiving rehabilitation had longer median survivals, fewer deaths from myelopathic complications, less pain 2 weeks after SEM treatment, lower depression scores, and higher satisfaction with life scores. In addition, among the patients who received rehabilitation, eight became independent for transfers (vs zero controls) and nine returned home (vs six controls). We conclude that directed rehabilitation reduced patients' pain levels and increased their mobility, survival, and life satisfaction.
\end{abstract}

Key words: cancer rehabilitation, depression, metastatic cancer, myelopathy, pain, paraplegia, satisfaction with life, spinal cord compression, spinal epidural metastasis, survival.

\section{INTRODUCTION}

A spinal epidural metastasis (SEM) compressing the spinal cord is the most common way that systemic cancer causes spinal cord dysfunction [1-2]. Many types of cancer metastasize to the spinal column, and 5 to 10 percent of people with cancer develop a symptomatic SEM [1-4]. SEMs are present in the autopsies of one-third of patients with cancer [5]. The annual incidence of SEM in the United States increased from 18,000 in 1987 to 25,000 in 1996 [2].

Among patients with spinal cord injury (SCI) (excluding patients with demyelinating diseases), 61 percent have traumatic SCI and 10 percent have SCI as a result of cancer [6]. During the first year after injury, the leading causes of death for people with traumatic SCI are respiratory diseases including pneumonia and infections originating in the urinary system or from pressure sores [7]. The mortality rate for nonambulatory patients with acute traumatic SCI is about 12 percent during the first 4 months

Abbreviations: ASIA = American Spinal Injury Association, BDI-II = Beck Depression Inventory-Second Edition, CI = confidence interval, LSCVAMC = Louis Stokes Cleveland Department of Veterans Affairs Medical Center, NSAID = nonsteroidal anti-inflammatory drugs, Rehab = rehabilitation, $\mathrm{RT}=$ radiation therapy, SCI = spinal cord injury, SEM = spinal epidural metastasis, SWLS = Satisfaction with Life Scale.

* Address all correspondence to Robert L. Ruff, MD, PhD; Neurology Service 127(W), Louis Stokes VA Medical Center, 10701 East Boulevard, Cleveland, OH 44106; 215-7913800, ext 5219; fax: 216-707-5934.

Email: robert.ruff1@va.gov

DOI: 10.1682/JRRD.2005.10.0168 
after injury [8]. One study found that the median survival of patients with SEM was 104 weeks for ambulatory patients and 6 weeks for nonambulatory patients [9]. In that study, 14 of the 30 nonambulatory patients (47\%) died of myelopathic complications, such as pneumonia, infected pressure sores, and urosepsis, which are the leading causes of death in the first year after traumatic SCI.

Several factors may contribute to the higher mortality rate of nonambulatory SEM patients compared with patients with traumatic SCI. Patients can die from direct complications of systemic cancer. In addition, the likelihood of adverse myelopathic complications increases in patients with SEM. In previous studies, the mean age of patients with SEM was about 40 years older than that of patients with traumatic SCI [8-9]. Furthermore, the coexistence of systemic cancer resulting in a catabolic state may increase the likelihood of complications of immobility and death [1]. Patient age and the presence of cancer are two factors that are not alterable. However, a third factor that is alterable is rehabilitation. Specialized rehabilitation is usually provided to patients with traumatic SCI but not to patients who develop SCI due to systemic cancer [6].

We performed a prospective evaluation of 12 consecutive male veterans who developed SEM and were unable to walk after completion of SEM treatment. Spinal cord medicine rehabilitation programs for people with traumatic SCI improve independence, self-perceived quality of life, and survival [7,10-11]. We developed a 2-week rehabilitation program that emphasized training in transfers, skin care, nutrition, and pulmonary exercise with incentive spirometry. Here, we report the outcomes of these 12 patients compared with a historical control group of the 30 nonambulatory patients from the aforementioned study of SEM [9]. The historical control group did not receive directed spinal cord rehabilitation. We examined patient survival, pain control, depression, mobility independence, frequency of returning home, and self-reported satisfaction with life. To our knowledge, this is the first study to evaluate spinal cord rehabilitation for people with paraplegia due to SEM.

\section{METHODS}

From July 2001 to September 2004, we prospectively evaluated 12 consecutive patients (Rehabilitation group, hereafter called the Rehab group) who presented to the Louis Stokes Cleveland Department of Veterans Affairs
Medical Center (LSCVAMC) with SEM and were unable to walk after completion of SEM treatment. Details of the SEM treatment with glucocorticoid medication (dexamethasone) and radiation therapy (RT) have been previously described [9]. The RT protocol and dexamethasone dose were the same for all subjects reported here. The SEM treatment protocol was similar to the protocols used in several prior studies of SEM [4,9,12-14]. Patients were considered ambulatory if they could walk without human assistance at least 50 feet without stopping. The 12 nonambulatory patients were offered the opportunity to engage in a 2-week rehabilitation program to help them adapt to their paraplegia. All patients accepted. This study was approved and continuously reviewed by the Quality Assurance Committee of the Neurology Department of Case Western Reserve University, the LSCVAMC Quality Assurance Service, the LSCVAMC Clinical Executive Committee, and the LSCVAMC Institutional Review Board.

\section{Rehabilitation Training}

The 2-week inpatient rehabilitation program emphasized patient and caregiver training on transfers, bowel and bladder care, incentive spirometry, nutrition, and skin care. Training was provided 6 days a week; each treatment day, patients received 2 hours of occupational therapy and 2 hours of training by a nurse that focused on transfers, wheelchair use, personal hygiene, incentive spirometry, skin care, and bowel and bladder management. In addition, patients received 30 minutes of physical therapy 6 days a week to maintain range of motion of paralyzed joints. This rehabilitation program included the essential elements of rehabilitation used for patients with paraplegia due to traumatic SCI [11]. Patients entered the rehabilitation program within 1 day of completing RT.

\section{Incentive Spirometry}

Each patient's nurse provided a 20-minute training in the use of incentive spirometry. The nurses encouraged the patients to increase their expiratory volumes and trained the caregivers to reinforce and, when needed, help patients perform incentive spirometry four times a day.

\section{Transfer and Unweighting Training}

Typically, most of the 2 hours of daily occupation therapy was devoted to transfer training and unweighting techniques. When a patient was unable to independently transfer from bed to chair and chair to commode, we trained the caregiver to use a lift to transfer the patient. 


\section{Skin Care and Bladder and Bowel Management}

Occupational therapists trained patients to use mirrors to facilitate skin inspection. Patients and caregivers also received about 2 hours of daily nursing instruction on skin care and bowel and bladder management. Nurses reinforced skin care and the transfer techniques learned in occupational therapy. Nurses taught patients and caregivers intermittent bladder catheterization techniques. Bowel care consisted of combined medication and mechanical techniques that facilitated bowel evacuation three times a week. Patients typically received $100 \mathrm{mg}$ of docusate sodium three times a day to soften their stool. The dose of stool softener was adjusted for adequate stool softening. The patient, caregiver, or both were trained to administer a minienema containing $283 \mathrm{mg}$ of docusate sodium along with digital stimulation to facilitate bowel evacuation. The 10 patients who could sit unassisted learned to complete bowel care on a raised commode seat that could be located above a conventional toilet. Nurses taught the caregivers of the other two patients to complete bowel care in bed. The in-bed bowel care training included instruction on the use of pads for preventing soiling of bedding.

\section{Dietary Counseling}

Each patient and caregiver in the Rehab group received two instructional sessions with a dietician to learn dietary manipulations to combat catabolism and to develop a diet that supported the patient's bowel management program.

\section{Historical Control Group}

The Rehab group was compared with a historical control group (hereafter called the No Rehab group) from a recently completed study of SEM treatment conducted at the LSCVAMC [9]. The No Rehab group included 30 veterans who were unable to walk after SEM treatment. The subjects in the No Rehab group did not receive spinal cord rehabilitation but did receive physical therapy 3 hours a week for at least 2 weeks. The physical therapy focused on range of limb motion and strengthening of residual lower-limb motor function. Measures of pain after completion of SEM treatment, depression severity, and satisfaction with life for the No Rehab group were previously collected but presented only in abstract form [15-17]. Demographics of the study and control groups are shown in Table $\mathbf{1}$.
Table 1.

Demographic comparison of study group that received 2 weeks of rehabilitation (Rehab, $n=12$ ) and control group that did not (No Rehab, $n=30$ ).

\begin{tabular}{lcr}
\hline \multicolumn{1}{c}{ Characteristic } & Rehab & No Rehab \\
\hline $\begin{array}{l}\text { Age (mean } \pm \text { standard error } \\
\text { of the mean) }\end{array}$ & $67.8 \pm 2.9$ & $69.1 \pm 1.6$ \\
Education, $n$ (\%) & & \\
High School & $6(50)$ & $13(43)$ \\
Some College & $3(25)$ & $9(30)$ \\
College Degree & $3(25)$ & $8(27)$ \\
Tumor Type, $n(\%)$ & & \\
Prostate & $5(42)$ & $13(43)$ \\
Lung & $5(42)$ & $12(40)$ \\
Other & $2(17)$ & $5(17)$ \\
Spinal Level, $n(\%)$ & & \\
Above T6 & $5(42)$ & $10(33)$ \\
T6 to T12 & $4(33)$ & $11(37)$ \\
Below T12 & $3(25)$ & $9(30)$ \\
ASIA Grade, $n(\%)$ & & \\
A & $6(50)$ & $11(37)$ \\
B & $6(50)$ & $12(40)$ \\
C & $0(0)$ & $6(20)$ \\
D & $0(0)$ & $1(3)$ \\
\hline *Pain
\end{tabular}

*Patient classified with American Spinal Injury Association (ASIA) grade D at onset of spinal epidural metastasis treatment and deteriorated to grade $\mathrm{B}$. $\mathrm{T}=$ thoracic.

Patients in both groups had had detailed neurological physical examinations before starting SEM treatment; the examinations included classification of the severity and level of the myelopathy according to the American Spinal Injury Association (ASIA) classification system [18]. Briefly, this classification system includes the following grades: $\mathrm{A}=$ complete myelopathy, $\mathrm{B}=$ sensory sparing without motor function, $\mathrm{C}=$ partial motor sparing with most tested muscles graded at $<3 / 5$ (not antigravity) on manual muscle testing, $\mathrm{D}=$ more complete motor sparing with most tested muscle graded at $>3 / 5$, and $E=$ no motor or sensory deficit. Patients in both groups were followed until their deaths. After discharge from inpatient care, patients were followed by telephone contacts every month and outpatient visits every 3 months.

\section{Subject Inclusion and Exclusion Criteria}

Our patient selection criteria were designed to include patients who had had similar SEM treatments. The inclusion criteria were (1) an SEM that was producing myelopathy and (2) an inability to walk after SEM treatment. The exclusion criteria were (1) refusal of SEM treatment, 
(2) cauda equina syndrome without myelopathy, (3) prior SEM that had produced myelopathy, and (4) surgical treatment of SEM. We excluded five patients who had developed paraplegia due to SEM (four who would have entered the No Rehab group and one who could have entered the Rehab group) because they refused SEM treatment. We excluded another four patients from the No Rehab group because they had had surgical spinal canal decompression because of the presence of bone fragments in the spinal canal. Excluding patients with bone fragments may have improved the survival of the No Rehab group because patients with bone fragments have very poor prognoses [19-20]. The patients who were excluded from this study had poor prognoses. Eight of the nine excluded patients would have entered the No Rehab group. Consequently, if the excluded patients had been included in the study, the No Rehab group would likely have had poorer outcomes.

\section{Outcome Measures}

The main outcome measure was patient survival. The secondary outcome measures were level of pain, ability to transfer, self-reported severity of symptoms of depression, self-reported satisfaction with life, frequency of achieving mobility independence, and frequency of returning home. Note that patients entered the Rehab group within 1 day of completing SEM treatment; therefore, measurements made 2 weeks after completion of SEM treatment in the No Rehab group were temporally comparable with measurements made at the completion of SEM treatment in the Rehab group.

\section{Depression and Satisfaction with Life}

Depression and satisfaction with life were assessed after completion of SEM treatment and completion of rehabilitation for the Rehab group or 2 weeks after completion of SEM treatment for the No Rehab group. Depression was assessed with the Beck Depression Inventory-Second Edition (BDI-II) [21]. The BDI-II is a 21-item self-report instrument that has been extensively validated. The BDI-II can be administered in less than 10 minutes. Each BDI-II item is scored on a four-point scale ranging from 0 to 3 . A total BDI-II score of 0 to 13 indicates minimal depression, 14 to 19 mild depression, 20 to 28 moderate depression, and 29 to 63 severe depression. Higher total scores indicate more severe depressive symptoms. The BDI-II is positively correlated with the Hamilton Depression Rating Scale (Pearson $r=$ 0.71). The test also has high 1-week test-retest reliability
(Pearson's $r=0.93$ ) [22] and high internal consistency ( $\alpha=0.91$ ) [23]. The BDI-II is routinely used for assessing self-reported symptoms of depression in persons with SCI [24-26] and persons with cancer that involves the central nervous system [27].

Self-perceived satisfaction with life was assessed with the Satisfaction with Life Scale (SWLS) [28-29]. The SWLS consists of five statements to which subjects respond using a seven-point scale, with 1 denoting strong disagreement and 7 strong agreement. A total SWLS score of 5 to 9 indicates extreme dissatisfaction, 10 to 14 dissatisfaction, 15 to 19 slight dissatisfaction, 20 neutral, 21 to 25 slight satisfaction, 26 to 30 satisfaction, and 31 to 35 extreme satisfaction. Coefficient $\alpha$ for the SWLS has been reported to be 0.87 , while test-retest reliability after a 2-month interval has been reported to be 0.82 [28]. The SWLS is valid and reliable for a variety of ages and applications. It demonstrates a high level of convergence on self- and peer-reported measures of subjective well-being and life satisfaction [30]. The SWLS is routinely used for evaluating self-reported satisfaction with life in persons with SCI [31-34]. The two groups' BDI-II and SWLS values are provided in Table 2.

\section{Pain}

We assessed pain levels before, immediately after, and 2 weeks after SEM treatment or completion of rehabilitation. The patients scored their highest pain levels experienced during the prior 24 hours on a numerical rating scale from 0 to 10 , with 0 indicating no pain and 10 unbearable pain [35]. Patients with pain levels $\leq 4$ were offered $650 \mathrm{mg}$ of acetaminophen four times a day for the pain. Patients with more intense pain were offered opioid

Table 2.

Beck Depression Inventory-Second Edition (BDI-II) and Satisfaction with Life Scale (SWLS) scores (mean \pm standard error of the mean). Measured just after completion of spinal epidural metastasis (SEM) treatment and after either 2 weeks directed rehabilitation (Rehab group, $n=12$ ) or 2 weeks after SEM treatment (No Rehab group, $n=30$ ).

\begin{tabular}{lll}
\hline \multicolumn{1}{c}{ Measure } & \multicolumn{1}{c}{ Rehab } & No Rehab \\
\hline BDI-II Post-SEM Treatment & $30.3 \pm 2.4$ & $29.6 \pm 2.4$ \\
BDI-II 2 wk Post-SEM Treatment & $13.2 \pm 3.5^{*}$ & $36.5 \pm 1.9$ \\
SWLS Post-SEM Treatment & $11.2 \pm 0.8$ & $10.8 \pm 0.6$ \\
SWLS 2 wk Post-SEM Treatment & $27.0 \pm 0.7^{*}$ & $11.1 \pm 0.7$ \\
\hline *Rehab had lower BDI-II and higher SWLS scores after rehabilitation than \\
before start of rehabilitation ( $p<0.001)$. At completion of rehabilitation, \\
Rehab had lower BDI-II and higher SWLS scores than No Rehab $(p<0.001)$. \\
\hline \hline
\end{tabular}


JRRD, Volume 44, Number 1, 2007

Table 4.

Statistical parameters for Group 1 (No Rehab, $n=30$ ) and Group 2 (Rehab, $n=12$ ).

\begin{tabular}{|c|c|c|c|c|c|}
\hline Analysis Variable & Group & $\begin{array}{l}\text { Mean } \pm \text { Standard } \\
\text { Error of the Mean }\end{array}$ & $\begin{array}{c}\text { Two-Sample } \\
\text { Wilcoxon Statistic }\end{array}$ & $\begin{array}{c}\text { Standardized } \\
\text { Wilcoxon Statistic }\end{array}$ & $\begin{array}{c}p \text {-Value, Wilcoxon } \\
\text { Test (Two-Sided) }\end{array}$ \\
\hline \multirow[t]{2}{*}{ Age } & 1 & $69.1 \pm 8.9$ & \multirow{2}{*}{239.5} & \multirow{2}{*}{-0.502} & \multirow{2}{*}{0.616} \\
\hline & 2 & $67.7 \pm 10.1$ & & & \\
\hline \multirow[t]{2}{*}{ BDI-II1 } & 1 & $29.6 \pm 13.2$ & \multirow{2}{*}{272.5} & \multirow{2}{*}{0.390} & \multirow{2}{*}{0.696} \\
\hline & 2 & $30.3 \pm 8.4$ & & & \\
\hline \multirow[t]{2}{*}{ BDI-II2 } & 1 & $36.5 \pm 10.8$ & \multirow{2}{*}{114.0} & \multirow{2}{*}{-4.001} & \multirow{2}{*}{$<0.001$} \\
\hline & 2 & $13.2 \pm 12.0$ & & & \\
\hline \multirow[t]{2}{*}{ PL1 } & 1 & $8.8 \pm 1.2$ & \multirow{2}{*}{265.5} & \multirow{2}{*}{0.204} & \multirow{2}{*}{0.838} \\
\hline & 2 & $8.9 \pm 1.2$ & & & \\
\hline \multirow[t]{2}{*}{ PL2 } & 1 & $6.4 \pm 1.6$ & \multirow{2}{*}{238.5} & \multirow{2}{*}{-0.547} & \multirow{2}{*}{0.584} \\
\hline & 2 & $6.2 \pm 0.9$ & & & \\
\hline \multirow[t]{2}{*}{ PL3 } & 1 & $6.4 \pm 1.8$ & \multirow{2}{*}{135.5} & \multirow{2}{*}{-3.467} & \multirow{2}{*}{$<0.001$} \\
\hline & 2 & $4.2 \pm 0.9$ & & & \\
\hline \multirow[t]{2}{*}{ SWLS1 } & 1 & $10.8 \pm 3.5$ & \multirow{2}{*}{263.5} & \multirow{2}{*}{0.140} & \multirow{2}{*}{0.889} \\
\hline & 2 & $11.2 \pm 2.7$ & & & \\
\hline \multirow[t]{2}{*}{ SWLS2 } & 1 & $11.1 \pm 3.7$ & \multirow{2}{*}{438.0} & \multirow{2}{*}{5.010} & \multirow{2}{*}{$<0.001$} \\
\hline & 2 & $27.0 \pm 2.5$ & & & \\
\hline
\end{tabular}

BDI-II1 = Beck Depression Inventory-Second Edition (BDI-II) post-SEM treatment and prerehabilitation, BDI-II2 = BDI-II postrehabilitation, PL1 = Pain Level (PL) pre-SEM treatment, PL2 = PL post-SEM treatment and prerehabilitation, PL3 = PL postrehabilitation, SEM = spinal epidural metastasis, SWLS1 = Satisfaction with Life Scale (SWLS) post-SEM treatment and prerehabilitation, SWLS2 = SWLS postrehabilitation.

Rehab group or 2 weeks after completion of SEM treatment for the No Rehab group, the number of subjects who were discharged to home were nine $(75 \%)$ for the Rehab group and six (20\%) for the No Rehab group ( $p<$ $0.01)$. No patient was discharged to home after the 2-week window after completion of SEM treatment. All patients who became independent for transfers were discharged to home. The risk ratio for being discharged to home for the Rehab group compared with the No Rehab group was 3.75 with a $95 \%$ CI of 2.31 to 6.09 .

\section{Depression}

After completion of SEM treatment, the No Rehab group patients were more likely to be diagnosed with clinical depression and treated with antidepressant medication by the physicians providing their day-to-day management. The managing physicians were unaware of the values of the data being collected. By 2 weeks after completion of SEM treatment, 26 patients in the No Rehab group $(86.7 \%)$ were clinically depressed and treated with antidepressant medications. Within the Rehab group, only one patient (8.3\%) was diagnosed with depression. The risk ratio for being clinically diagnosed with depression among No Rehab patients compared with Rehab patients was 10.4 with a $95 \%$ CI of 5.81 to 18.6. For comparison,

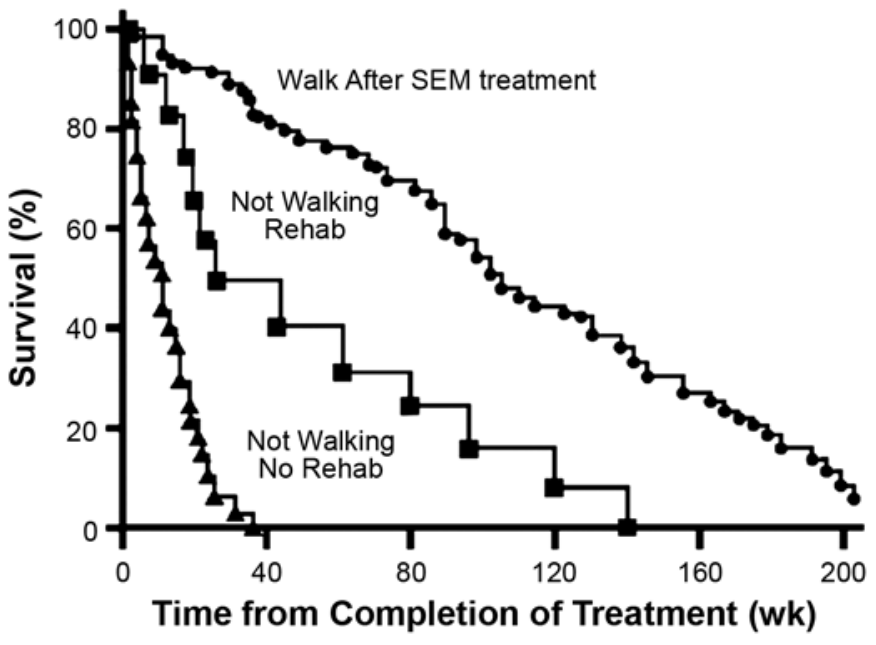

Figure.

Rehabilitation (rehab) prolongs life of nonambulatory patients with spinal epidural metastasis (SEM). Comparison of survival after completion of SEM treatment. Curves shown for patients who could not walk after completion of SEM treatment and did not receive rehab ( $\boldsymbol{\Delta}$, Not Walking No Rehab), patients who could not walk after completion of SEM treatment and received 2 wk rehab ( $\mathbf{\square}$, Not Walking Rehab), and patients who could walk after completion of SEM treatment $(\bullet$, Walk after SEM treatment). Source (Not Walking No Rehab and Walk After SEM treatment groups data): Zaidat OO, Ruff RL. Treatment of spinal epidural metastasis improves patient survival and functional state. Neurology. 2002;58(9):1360-66. [PMID: 12011281] 
8.26 percent of the 109 ambulatory patients from the prior SEM study were clinically depressed $(p<0.001)$ [9].

The BDI-II scores showed that the severity of depression at the completion of SEM treatment was similar for the Rehab and No Rehab groups (Table 2). However, at 2 weeks after completion of SEM treatment, the BDI-II scores supported the clinical perception of more severe depression among the No Rehab patients. The mean BDI-II score after the 2-week rehabilitation for the Rehab group corresponded to minimal to mild depression. In contrast, the mean BDI-II score for the No Rehab group indicated severe depression.

\section{SWLS Scores}

At the completion of SEM treatment, the two groups had similar SWLS scores (Table 2). The scores for both groups at the completion of SEM treatment indicated that the patients were dissatisfied with life. The SWLS scores for the No Rehab group had not improved at 2 weeks after completion of SEM treatment. In contrast, the Rehab group had higher SWLS scores after completion of rehabilitation than at the start of rehabilitation $(p<$ 0.001). At completion of rehabilitation, the Rehab group had higher SWLS scores compared with the No Rehab group $(p<0.001)$. The Rehab group's mean SWLS score indicated satisfaction with life.

\section{Pain}

At the onset of SEM treatment, patients rated their highest pain levels within the past 24 hours on a 0 to 10 pain scale. Both patient groups had similar, high levels of pain before SEM treatment (Table 3). Both patient groups reported decreased pain, to a similar degree, in association with SEM treatment. However, pain levels differed significantly between the two groups 2 weeks after completion of SEM treatment (Table 3). Pain levels for the No Rehab group did not change during the 2 weeks after completion of SEM treatment. In contrast, after completion of the rehabilitation program, the Rehab group had lower pain levels than before the rehabilitation program and lower pain levels than the No Rehab group.

The lower pain levels reported by patients in the Rehab group were not due to more aggressive medical treatment of pain. To compare the amount of pain medications consumed by the patients, we used standard pain medication conversion formulas to convert total daily intake of opioids and NSAID to equivalent doses of morphine sulfate. The Rehab group consumed 35 percent of the dose of opioids and 32 percent of the dose of total pain medication (opioid + NSAID) compared with the No Rehab group.

\section{DISCUSSION}

In this study, the outcomes of the two subject groups were significantly different for the main outcome measure and all the secondary outcome measures. The patients with paraplegia due to SEM who received a 2-week course of directed rehabilitation lived longer (Figure), were more likely to be independent for transfers, were more likely to return home, had lower BDI-II scores that were associated with a lower prevalence of clinical depression (Table 2), had lower pain scores (Table 3), and had higher SWLS scores that indicated that, as a group, they were satisfied with life (Table 2). We suggest that the reduced likelihood of clinical depression, lower BDI-II scores, and higher SWLS scores for the Rehab group resulted in part from these patients' reduced pain and greater independence.

The Rehab and No Rehab groups were not evaluated simultaneously. We are unaware of any changes in the SEM treatment between the period in which the No Rehab patients were treated and the period in which the Rehab patients were treated. However, some differences in the outcomes of groups may have resulted from unrecognized changes in patient care rather than from the rehabilitation provided to the Rehab group.

The rehabilitation program that we developed was abbreviated compared with programs provided to people with traumatic SCI because we recognized that our subjects' life expectancies were shortened by their systemic cancers. The customized 2-week protocol excluded some aspects of spinal cord rehabilitation programs designed for people with traumatic SCI. We did not include driver training, recreational therapy, pool therapy, or physical therapy directed at increasing lower-limb strength for eventual standing and walking training. In addition to shorter life expectancies, the subjects in our study were elderly and therefore we excluded therapy designed to help people stand and walk after SCI. One author's personal experience with spinal cord rehabilitation has been that, for similar severity of myelopathy, elderly people are less likely to regain standing and ambulation skills and often do not have the cardiovascular endurance required to complete physical therapy designed to help the patient return to standing and walking. An additional 
factor considered was that the nonambulatory subjects in this study had severe myelopathy. Prognosis for recovery of function is associated with ASIA classification for patients with traumatic SCI [43-44]. Patients who have paraplegia with ASIA grades A or B are unlikely to recover walking. We recently analyzed the prognostic value of ASIA grades in patients with SEM [45]. We found that patients who had myelopathy with ASIA grades A, B, or C and were unable to walk at the completion of SEM treatment did not recover walking ability later. All the patients in the Rehab group were ASIA grades A or B. Hence, we felt that excluding rehabilitation aimed at recovery of ambulation was justified because of the time required for such training and the low likelihood of success.

The survival of the Rehab group was intermediate when compared with the No Rehab group and the ambulatory group of patients from the prior study of SEM conducted at the LSCVAMC (Walk After SEM treatment, Figure) [9]. Several prior studies of SEM suggested that patients who walk after SEM treatment live longer $[9,13,19-20]$. Some of the difference in survival for ambulatory versus nonambulatory patients may have resulted from differences in the aggressiveness of individual cancers [1-2].

The longer survival of the Rehab group compared with the No Rehab group (Figure) was due in part to the elimination of deaths from complications of myelopathy. None of the patients in the Rehab group died of complications of myelopathy, whereas 47 percent of the patients in the No Rehab group did. In addition, the shorter survival of the No Rehab group compared with the Rehab group may have been related to increased depression among the No Rehab patients. Increased depression, determined by the BDI-II, was associated with shorter survival in people with central nervous system cancer [27]. Depression can affect the survival of people with cancer in several possible ways. A person with cancer and depression may concede and surrender to the cancer, including stopping any treatments that may alter cancer progression [27]. Depression may be more than a purely psychological reaction to the cancer. Physiological changes induced by depression, including disrupted hypothalamic-pituitary function, changed cytokine activity, and altered fatty acid and phospholipid metabolism, can alter the body's responses to cancer [46-49]. Depression is associated with elevated cortisol levels, and effective treatment of depression will reduce cortisol levels and lengthen survival [50-51].
Based upon the findings of this study, the LSCVAMC Spinal Cord Injury Unit has adopted a policy of offering directed rehabilitation to patients unable to walk after SEM treatment. This policy was approved by the LSCVAMC Quality Assurance Service and the LSCVAMC Clinical Executive Committee. The Rehab group included only 12 patients. Consequently, other facilities may wish to consider data from a larger study group before adopting a similar policy. For those who wish to provide rehabilitation for cancer patients who develop SEM, we suggest the following three criteria for selecting the patients who will benefit most: (1) the patients and their support network should be accepting and willing to participate in a rehabilitation program, (2) the state of the primary malignancy should permit an estimated survival of 6 months or more, and (3) resources should be available for educating patients and their caregivers on transfers, skin care, incentive spirometry, and nutrition. Future studies on rehabilitation for nonambulatory SEM patients might consider ways to provide rehabilitation training on an outpatient basis and determine whether rehabilitation should target a subset of nonambulatory SEM patients.

\section{CONCLUSIONS}

The findings in this study suggest that provision of a 2-week rehabilitation program increased survival. At the end of the rehabilitation program, patients had less pain and depression and improved satisfaction with life. We believe that the differences in outcomes resulted from the 2-week rehabilitation program. However, other factors possibly could have contributed to the differences in the outcomes of the two study groups. The Rehab and No Rehab patients were evaluated at different times. While we are unaware of systematic changes in patient care, unrecognized differences in the experiences of the two groups could possibly have contributed to the differences in outcomes.

A more convincing protocol for evaluating the effect of rehabilitation on people unable to walk after completing SEM treatment would be a randomized controlled multicenter clinical trial. Hopefully, the findings presented here will stimulate and justify a randomized clinical trial of rehabilitation for people who are nonambulatory due to an SEM. 


\section{ACKNOWLEDGMENTS}

Portions of this work were presented at the 2004 and 2005 meetings of the American Academy of Neurology and the 2003 meeting of the American Neurological Association.

This material was the result of work supported by a grant from the Department of Veterans Affairs, Office of Research and Development, Rehabilitation Research and Development Service.

The authors have declared that no competing interests exist.

\section{REFERENCES}

1. Posner JB. Back pain and epidural spinal cord compression. Med Clin North Am. 1987;71(2):185-205. [PMID: 2950289]

2. Lada R, Kaminski HJ, Ruff RL. Metastatic spinal cord compression. In: Vecht C, editor. Neuro-oncology part 3. Neurological disorders in systemic cancer. Amsterdam (the Netherlands): Elsevier Biomedical Publishers; 1997. p. 167-89.

3. Barron KD, Hirano A, Araki S, Terry RD. Experiences with metastatic neoplasms involving the spinal cord. Neurology. 1959;9(2):91-106. [PMID: 13622902]

4. Gilbert RW, Kim JH, Posner JB. Epidural spinal cord compression from metastatic tumor: Diagnosis and treatment. Ann Neurol. 1978;3(1):40-51. [PMID: 655653]

5. Bach F, Larsen BH, Rohde K, Borgesen SE, Gjerris F, Boge-Rasmussen T, Agerlin N, Rasmusson B, Stjernholm P, Sorensen PS. Metastatic spinal cord compression. Occurrence, symptoms, clinical presentations and prognosis in 398 patients with spinal cord compression. Acta Neurochir (Wien). 1990;107(1-2):37-43. [PMID: 2096606]

6. McKinley WO. Nontraumatic spinal cord injury: Etiology, incidence and outcome. In: Kirshblum S, Campagnolo DI, DeLisa JA, editors. Spinal cord medicine. Philadelphia (PA): Lippincott Williams \& Wilkins; 2002. p. 471-79.

7. DeVivo MJ. Epidemiology of traumatic spinal cord injury. In: Kirshblum S, Campagnolo DI, DeLisa JA, editors. Spinal cord medicine. Philadelphia (PA): Lippincott Williams \& Wilkins; 2002. p. 69-81.

8. Bracken MB, Freeman DH Jr, Hellenbrand K. Incidence of acute traumatic hospitalized spinal cord injury in the United States, 1970-1977. Am J Epidemiol. 1981;113(6): 615-22. [PMID: 7234849]

9. Zaidat OO, Ruff RL. Treatment of spinal epidural metastasis improves patient survival and functional state. Neurology. 2002;58(9):1360-66. [PMID: 12011281]

10. DeLisa JA, Hammond MC. The history of the subspecialty of spinal cord injury medicine. In: Kirshblum S, Campag- nolo DI, DeLisa JA, editors. Spinal cord medicine. Philadelphia (PA): Lippincott Williams \& Wilkins; 2002. p. 1-4.

11. Kirshblum S, Ho CH, House JG, Druin E, Nead C, Drastal S. Rehabilitation of spinal cord injury. In: Kirshblum S, Campagnolo DI, DeLisa JA, editors. Spinal cord medicine. Philadelphia (PA): Lippincott Williams \& Wilkins; 2002. p. 275-98.

12. Greenberg HS, Kim JH, Posner JB. Epidural spinal cord compression from metastatic tumor: Results with a new treatment protocol. Ann Neurol. 1980;8(4):361-66. [PMID: 7436380]

13. Ruff RL, Lanska DJ. Epidural metastases in prospectively evaluated veterans with cancer and back pain. Cancer. 1989;63(11):2234-41. [PMID: 2524249] Erratum in: Cancer. 1990;66(5):935.

14. Smith EM, Hampel N, Ruff RL, Bodner DR, Resnick MI. Spinal cord compression secondary to prostate carcinoma: Treatment and prognosis. J Urol. 1993;149(2):330-33. [PMID: 8426413]

15. Adamson VM, Fredrickson E, Ruff RL. Spinal epidural metastases (SEM): Rehabilitation increases survival and reduces pain [abstract]. Ann Neurol. 2003;54:S63-64.

16. Adamson VM, Ruff SS, Zaidat OO, Ruff RL. Benefits of providing directed rehabilitation to patients with paraplegia after spinal cord compression caused by epidural metastatic cancer [abstract]. Neurology. 2004;62:A458-59.

17. Adamson VW, Ruff SS, Ruff RL. Directed rehabilitation reduces depression and pain and increases independence and life satisfaction for patients with paraplegia due to epidural metastatic spinal cord compression [abstract]. Neurology. 2005;64:A405.

18. Kirshblum S, Donovan WH. Neurological assessment and classification of traumatic spinal cord injury. In: Kirshblum S, Campagnolo DI, DeLisa JA, editors. Spinal cord medicine. Philadelphia (PA): Lippincott Williams \& Wilkins; 2002. p. 82-95.

19. Zelefsky MJ, Scher HI, Krol G, Portenoy RK, Leibel SA, Fuks ZY. Spinal epidural tumor in patients with prostate cancer. Clinical and radiographic predictors of response to radiation therapy. Cancer. 1992;70(9):2319-25.

[PMID: 1394060]

20. Tomita T, Galicich JH, Sundaresan N. Radiation therapy for spinal epidural metastases with complete block. Acta Radiol Oncol. 1983;22(2):135-43. [PMID: 6310968]

21. Beck AT, Steer RA, Brown GK. Beck Depression Inventory. 2nd ed. San Antonio (TX): The Psychological Corporation; 1996. p. 128.

22. Beck AT, Steer RA, Brown GK. Manual for the Beck Depression Inventory-II. San Antonio (TX): The Psychological Corporation; 1996.

23. Beck AT, Steer RA, Ball R, Ranieri WF. Comparison of Beck Depression Inventories-IA and -II in psychiatric outpatients. J Pers Assess. 1996;67(3):588-97. [PMID: 8991972] 
24. Tirch D, Radnitz CL, Bauman WA. Depression and spinal cord injury: A monozygotic twin study. J Spinal Cord Med. 1999;22(4):284-86. [PMID: 10751132]

25. Kennedy P, Rogers BA. Anxiety and depression after spinal cord injury: A longitudinal analysis. Arch Phys Med Rehabil. 2000;81(7):932-37. [PMID: 10896007]

26. Osteraker AL, Levi R. Indicators of psychological distress in postacute spinal cord injured individuals. Spinal Cord. 2005;43(4):223-29. [PMID: 15583705]

27. Mainio A, Hakko H, Timonen M, Niemela A, Koivukangas J, Rasanen P. Depression in relation to survival among neurosurgical patients with a primary brain tumor: A 5-year follow-up study. Neurosurgery. 2005;56(6):1234-41.

[PMID: 15918939]

28. Diener E, Emmons RA, Larsen RJ, Griffin S. The Satisfaction with Life Scale. J Pers Assess. 1985;49(1):71-75. [PMID: 16367493]

29. Pavot W, Diener E. Review of the Satisfaction with Life Scale. Psychol Assess. 1993;5(2):164-72.

30. Pavot W, Diener E, Colvin CR, Sandvik E. Further validation of the Satisfaction with Life Scale: Evidence for the cross-method convergence of well-being measures. J Pers Assess. 1991;57(1):149-61. [PMID: 1920028]

31. Consortium for Spinal Cord Medicine. Outcomes following traumatic spinal cord injury: Clinical practice guidelines for health care professionals. Washington (DC): Paralyzed Veterans of America; 1999.

32. Rintala DH, Robinson-Whelen S, Matamoros R. Subjective stress in male veterans with spinal cord injury. J Rehabil Res Dev. 2005;42(3):291-304. [PMID: 16187242]

33. Johnston MV, Diab ME, Kim SS, Kirshblum S. Health literacy, morbidity, and quality of life among individuals with spinal cord injury. J Spinal Cord Med. 2005;28(3):230-40. [PMID: 16048141]

34. Richards JS, Bombardier CH, Tate D, Dijkers M, Gordon W, Shewchuk R, DeVivo MJ. Access to the environment and life satisfaction after spinal cord injury. Arch Phys Med Rehabil. 1999;80(11):1501-6. [PMID: 10569447]

35. Kremer E, Atkinson JH, Ignelzi RJ. Measurement of pain: Patient preference does not confound pain measurement. Pain. 1981;10(2):241-48. [PMID: 7267140]

36. Gammaitoni AR, Fine P, Alvarez N, McPherson ML, Bergmark S. Clinical application of opioid equianalgesic data. Clin J Pain. 2003;19(5):286-97. [PMID: 12966254]

37. Machin D, Gardner MJ. Time to event studies. In: Altman DG, Machin D, Bryant TN, Gardner MJ, editors. Statistics with confidence. 2nd ed. Bristol (England): BMJ Press; 2000. p. 93-194.

38. Parmar MK, Machin D. Survival analysis: A practical approach. New York (NY): John Wiley; 1995. p. 26-40, 115-42.

39. Peto J. The calculation and interpretation of survival curves. In: Buyse ME, Staquet MJ, Sylvester RJ, editors.
Cancer clinical trials: Methods and practice. Oxford (England): Oxford University Press; 1984. p. 361-80.

40. Miller RG. Beyond ANOVA: Basics of applied statistics. New York (NY): John Wiley; 1986.

41. Hollander M, Wolfe DA. Nonparametric statistical inference. 2nd ed. New York (NY): John Wiley \& Sons; 1999. p. 68-75.

42. Morris JA, Gardner MJ. Epidemiological studies. In: Altman DG, Machin D, Bryant TN, Gardner MJ, editors. Statistics with confidence. 2nd ed. Bristol (England): BMJ Press; 2000. p. 57-72.

43. DiTunno JF Jr. The John Stanley Coulter Lecture. Predicting recovery after spinal cord injury: A rehabilitation imperative. Arch Phys Med Rehabil. 1999;80(4):361-64. [PMID: 10206595]

44. Marino RJ, DiTunno JF Jr, Donovan WH, Maynard F Jr. Neurological recovery after traumatic spinal cord injury: Data from the Model Spinal Cord Injury Systems. Arch Phys Med Rehabil. 1999;80(11):1391-96. [PMID: 10569432]

45. Ruff RL, Zaidat OO, Adamson VW. Correlation between ASIA level and outcomes of patients with spinal epidural metastases. J Spinal Cord Medicine. 2004;27:408-9.

46. Evans DL, Charney DS. Mood disorder and medical illness: A major public health problem. Biol Psychiatry. 2003; 54(3):177-80. [PMID: 12893090]

47. Horrobin DF, Bennett CN. Depression and bipolar disorder: Relationships to impaired fatty acid and phospholipid metabolism and to diabetes, cardiovascular disease, immunological abnormalities, cancer, ageing and osteoporosis. Possible candidate genes. Prostaglandins Leukot Essent Fatty Acids. 1999;60(4):217-34. [PMID: 10397403]

48. Spiegel D, Giese-Davis J. Depression and cancer: Mechanisms and disease progression. Biol Psychiatry. 2003; 54(3):269-82. [PMID: 12893103]

49. Katon WJ. Clinical and health services relationships between major depression, depressive symptoms, and general medical illness. Biol Psychiatry. 2003;54(3):216-26. [PMID: 12893098]

50. Cruess DG, Antoni MH, McGregor BA, Kilborne KM, Boyers AE, Alferi SM, Carver CS, Kumar M. Cognitivebehavioral stress management reduces serum cortisol by enhancing benefit finding among women being treated for early stage breast cancer. Psychosom Med. 2000;62(3): 304-8. [PMID: 10845343]

51. Van der Pompe G, Duivenvoorden HL, Antoni MH, Visser A, Heijnen CJ. Effectiveness of a short-term group psychotherapy program on endocrine and immune function in breast cancer patients: An exploratory study. J Psychosom Res. 1997;42(5):453-66. [PMID: 9194018]

Submitted for publication October 29, 2005. Accepted in revised form January 24, 2007. 